Analysis of the early stage of thermal runaway

Dold, J W

1985

MIMS EPrint: 2007.85

Manchester Institute for Mathematical Sciences

School of Mathematics

The University of Manchester

\footnotetext{
Reports available from: http://eprints.maths.manchester.ac.uk/

And by contacting: The MIMS Secretary

School of Mathematics

The University of Manchester

Manchester, M13 9PL, UK
} 


\title{
ANALYSIS OF THE EARLY STAGE OF THERMAL RUNAWAY
}

\author{
By J. W. DOLD $\dagger$ \\ (College of Aeronautics, Cranfield Institute of Technology, Cranfield, \\ Bedfordshire MK43 0AL)
}

[Received 4 November 1983. Revise 2 August 1984]

\begin{abstract}
SUMMARY
It is shown that a variable grouping containing a logarithmic time-dependent factor is required for the development of a coordinate-perturbation expansion which realistically describes the spatially varying thermal-runaway process. The resulting solution is in good agreement with careful numerical computations. It describes a self-focussing temperature growth, the form and behaviour of which are remarkably independent of both the conditions leading to thermal runaway and the topology of the thermal-runaway region. The detailed solution also reveals an underlying structure in the temperature development, in which a strongly supercritical thermal runaway (where the relative effects of conduction are initially small) is found to be very much like a less strongly supercritical, but more highly-developed, thermal runaway. In this development the local rate of self-heating accelerates dramatically while, in comparison, the conductive impediment to the temperature growth diminishes towards zero. Attempts to develop a solution using a variable grouping without the logarithmic factor are shown to produce results which are unsuitable for describing inhomogeneous thermal runaway.

Some numerically computed results are presented, detailing the Ignition Kernel formed as a result of supercritical thermal runaway in a fixed-temperature symmetric container.
\end{abstract}

\section{Introduction}

THE essential nature of spatially varying thermal runaway can be studied using the equation

$$
\phi_{t}=\nabla^{2} \phi+\lambda(r, t) e^{\phi},
$$

where $\phi$ asymptotically represents the temperature in a large-activationenergy, small-reactant-depletion model. The function $\lambda$ represents the effect on the reaction rate of the mixture of chemical species and may be taken to be a known, possibly uniform, regular function of space and time.

Assuming that $\phi$ is not homogeneous in space, the most vital property of equation (1) is the possible development of a rapidly accelerating growth in temperature, concentrated about a point, line or surface. Figure 1 provides an illustration of this. Such behaviour arises when conditions are suitably 1TW

† Present address: School of Mathematics, University of Bristol, University Walk, Bristol BS8

[Q. Jl Mech. appl. Math., Vol. 38, Pt. 3, 1985] 

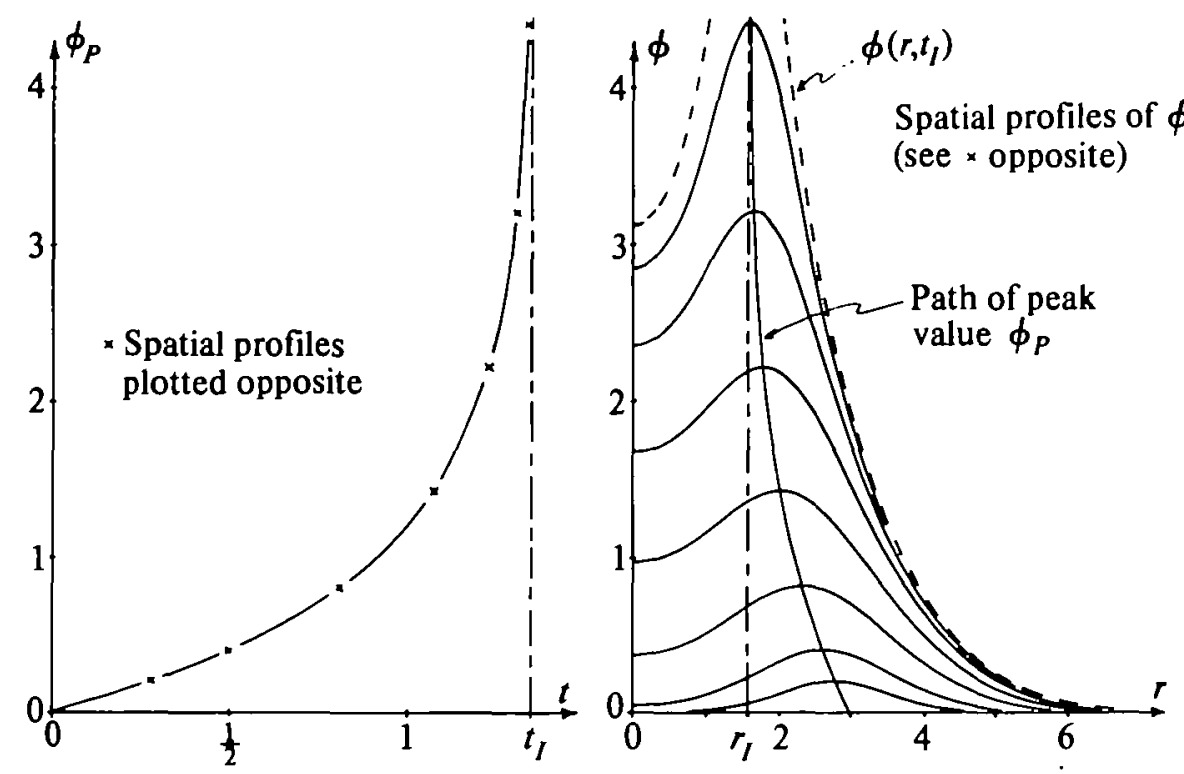

Fio. 1. A sample of the way in which $\phi$ varies

supercritical; factors impeding the growth of $\phi$, such as heat losses through the walls of a containing vessel $(1,2)$ or 'starvation' of the reaction by the diffusive dilution of a reactant (3), are insufficient to counterbalance the Arrhenius exponential dependence of the reaction rate on $\phi$. In fact $\phi$ becomes singular, approaching infinity at an 'induction position' $r_{I}$ as $t$ approaches an 'induction time' $t_{\mathrm{I}}$.

The asymptotic representation of the temperature by $\phi$, of course, breaks down at this stage; equation (1) does not model the consumption of chemical species which becomes more significant as $\phi$ grows large. In the early stages, however, equation (1) is a good representation which can be used to reveal important aspects of the basic nature of the thermal-runaway process. The full thermal development will be the subject of a subsequent paper (4).

As $\phi$ increases, the distribution of temperature around its sharply rising peak value becomes more-and-more localized, with the most vigorously increasing reaction intensities occupying a correspondingly shrinking region of space as the induction time is approached. A 'kernel' of self-igniting reactants is formed. The natural way of approaching this problem analytically is to develop a coordinate-perturbation solution using a suitable variable grouping to represent this spatial concentration of the effects of the reaction. Efforts have been made in this direction $(5,6)$ by using a variable grouping which differs significantly from the one which is found to be necessary in the following analysis. For the sake of clarity a distinction 
between these approaches will be made by referring to the former as characterizing a 'Hot Spot' (the term used in $(5,6)$ ) while the description 'Ignition Kernel' (as in (7)) will be used in conjunction with the analysis developed here. The terms, of course, refer to exactly the same phenomenon which both approaches are intended to model.

It is demonstrated that the required Ignition-Kernel variable grouping takes the form

$$
\rho=\frac{r-r_{I}}{\left(t_{I}-t\right)^{\frac{1}{2}}\left[\alpha-\ln \left(t_{I}-t\right)\right)^{\frac{1}{3}}},
$$

where $\alpha$ is open to choice and may be 'weakly' time dependent. This contrasts with the Hot-Spot variable grouping in which the logarithmic factor is absent. It is found that non-trivial solutions obtained using the Hot-Spot grouping are either singular at the induction position or vary too rapidly in space to be capable of being matched with temperature developments in relatively cooler regions. In analysing an equation related in type to equation (1), but with a quadratic rather than exponential reaction-rate term, Lacey (8) mentions that the Hot-Spot grouping appears to lead to no suitable solution. Solutions obtained using the Ignition-Kernel grouping (2) are found to agree well with careful numerical solutions of equation (1). Matching with thermal behaviour 'outside' the Ignition Kernel, in regions relatively much cooler than the hottest temperature at some stage, is achieved through an intermediate expansion.

In order to simplify the problem while retaining some generality it is convenient to use the following form of equation (1):

$$
\phi_{t}=\phi_{r r}+\frac{n}{r} \phi_{r}+\lambda(r, t) e^{\phi}
$$

where

$$
n= \begin{cases}0, & \text { for variation in only one Cartesian dimension, } \\ 1, & \text { for cylindrical symmetry, } \\ 2, & \text { for spherical symmetry. }\end{cases}
$$

In cases for which thermal runaway occurs, it may be taken that an induction position $r_{I}$ and an induction time $t_{I}$ exist such that

$$
\phi\left(r_{I}, t_{I}\right)=\infty .
$$

For simplicity it may also, quite generally, be assumed that the scalings of $r$ and $t$ are suitably normalized to make

$$
\lambda\left(r_{I}, t_{I}\right)=1 .
$$




\section{Vartable grouping characterizing thermal runaway}

A range of variable groupings can be conveniently examined by means of the scaling function $\sigma(\xi)$ introduced in the following definitions:

$$
\left.\begin{array}{c}
\xi=-\ln \left(t_{I}-t\right), \\
\eta=\frac{r-r_{I}}{\left[e^{-\xi} \sigma(\xi)\right]^{t}}, \\
\Phi(\xi, \eta)=\phi-\xi .
\end{array}\right\}
$$

It is found that, at its peak value, $\phi$ grows essentially like the parameter $\xi$ as $t \rightarrow t_{I}$ so that, with $\sigma$ chosen correctly, the dependent variable $\Phi$ introduced above can be expected to be primarily a non-trivial order-one function of $\eta$ in the thermal-runaway region. In terms of $\xi$ and $\eta, \Phi$ satisfies the equation

$$
\Phi_{\xi}+1+\frac{\eta}{2}\left(1-\frac{\dot{\sigma}}{\sigma}\right) \Phi_{\eta}=\sigma^{-1}\left\{\Phi_{\eta \eta}+\frac{n\left[e^{-\xi} \sigma\right]^{\frac{1}{2}}}{r_{I}+\left[e^{-\xi} \sigma\right]^{\frac{1}{2}} \eta} \Phi_{\eta}\right\}+\lambda e^{\Phi}
$$

(where ' signifies ordinary differentiation with respect to the appropriate independent variable). This equation can be used to examine the influence of the choice of the scaling function $\sigma$ on the possible forms of asymptotic behaviour of $\Phi$ as a function of $\xi$ and $\eta$.

The case of $\sigma$ being asymptotically small as $\xi$ tends to infinity can be dismissed since, with $\Phi$ of order one as anticipated, this would emph-size the conductive terms (on the right-hand side) of equation (7) whereas the essential nature of the thermal runaway lies with the exponential dependence of the reaction rate on $\Phi$. The cooling effect of conduction cannot be overwhelmingly dominant in the process. If $\sigma$ is of order one, the definition of $\eta$ amounts to the definition of the Hot-Spot variable grouping. This is examined in the next section.

If, on the other hand, $\sigma$ is asymptotically large as $\xi$ grows large, then it may be possible to develop an asymptotic expansion for $\Phi$, as $t$ tends to $t_{I}$, in the form

$$
\Phi \sim \Phi_{0}(\eta)+\ldots+\sigma^{-1} \Phi_{\sigma}(\eta)
$$

Any terms of order strictly between one and $\sigma^{-1}$ will not be considered in this section, the purpose of which is primarily to demonstrate the feasibility of such an asymptotic solution for a suitable choice of $\sigma$. The form that this choice must take is also demonstrated. A fuller development of the expansion is given in section 4.

To first order, equation (7) shows that $\Phi_{0}$ should satisfy the equation

$$
1+\frac{1}{2} \eta \dot{\Phi}_{0}=e^{\Phi_{0}}
$$

so that

$$
\Phi_{0}=-\ln \left[1+\frac{1}{4} \eta^{2}\right]
$$


where, by hypothesis, $\Phi_{0}$ has its non-trivial solution and where, for simplicity, the constant of integration $k$, in the solution $\Phi_{0}=-\ln \left[1+\frac{1}{4} k \eta^{2}\right]$, has been set to unity by exploiting a degree of freedom afforded by the choice of $\sigma$ in the definition of $\eta$. Proceeding to the term of order $\sigma^{-1}$, equations (7) to (9) give the following equation for $\Phi_{\sigma}$ :

$$
\Phi_{\sigma}-\left(1+\frac{\eta^{2}}{4}\right) \frac{\eta}{2} \dot{\Phi}_{\sigma}=\frac{1+n_{I}}{2}+[\dot{\sigma}]^{*} \frac{\eta^{2}}{4}-\frac{\frac{1}{4} \eta^{2}}{1+\frac{1}{4} \eta^{2}},
$$

where

$$
n_{I}= \begin{cases}0, & r_{I} \neq 0 \\ n, & r_{I}=0\end{cases}
$$

and

$$
[\dot{\sigma}]^{*}=\lim _{\xi \rightarrow \infty} \dot{\sigma}(\xi) .
$$

The notation [ $]^{*}$ will have the same meaning throughout this paper. The parameter $n_{I}$ distinguishes between the three different possible topologies of the induction position $r=r_{I}$. When $n_{I}$ is zero the induction position represents a surface, which can be flat, cylindrical or spherical depending on the value of $n$. When $n_{I}$ is unity it represents a line, and when $n_{I}$ has the value two it represents a single point. Solving for $\Phi_{\sigma}$ gives

$$
\begin{aligned}
\Phi_{\sigma}= & \frac{1+n_{I}}{2}+\frac{\frac{1}{4} \eta^{2}}{1+\frac{1}{4} \eta^{2}}\left[\text { const. }-[\dot{\sigma}]^{*} \ln \left(1+\frac{1}{4} \eta^{2}\right)\right]+ \\
& +\left(1-[\dot{\sigma}]^{*}\right) \frac{\frac{1}{4} \eta^{2}}{1+\frac{1}{4} \eta^{2}} \ln \left(\frac{\eta^{2}}{1+\frac{1}{4} \eta^{2}}\right)
\end{aligned}
$$

Although $\Phi_{\sigma}$ is continuous, if $1-[\dot{\sigma}]^{*}$ does not vanish then the last term in the solution (11) for $\Phi_{\sigma}$ has a singular second derivative with respect to $\eta$. This would make the term of order $\sigma^{-2}$ in the expansion for $\Phi$ singular at $\eta=0$, thereby invalidating the expansion. Thus $\sigma$ must satisfy the condition

$$
[\dot{\sigma}]^{*}=1 \text {, }
$$

from which it can be deduced that

$$
\sigma(\xi)=\xi+o(\xi)
$$

as $\xi \rightarrow \infty$, apart from the multiplication by an arbitrary constant which was implicity set to unity by the choice of the solution (9) for $\Phi_{0}$.

Although the $o(\xi)$ term in (13) is open to choice, its effect on the resulting scaling for $\eta$ in the definition (6) is asymptotically small as $\xi \rightarrow \infty$. Essentially the same variable grouping therefore arises and can be seen to correspond to the Ignition-Kernel grouping $\rho$ defined in (2), provided only that $\alpha$ is small compared with $\left|\ln \left(t_{I}-t\right)\right|$ as $t$ tends to $t_{\mathrm{r}}$. 


\section{Consideration of the Hot-Spot variable grouping}

With $\sigma$ defined as unity, $\eta$ takes on the form of the Hot-Spot variable grouping

$$
s=\frac{r-r_{I}}{\left(t_{I}-t\right)^{\frac{1}{2}}},
$$

and if $\phi$ is assumed to behave in accordance with the asymptotic form

$$
\phi \sim-\ln \left(t_{I}-t\right)+g(s),
$$

then it can be seen that $g(s)$ should satisfy the nonlinear second-order ordinary differential equation,

$$
\tilde{\mathrm{g}}+\left(\frac{n_{I}}{s}-\frac{s}{2}\right) \dot{\mathrm{g}}+e^{\mathrm{g}}-1=0 .
$$

Indeed, if $g$ satisfied this equation, then in those cases for which $\lambda(r, t)=1$ with $r_{I}=0$ when $n>0$, equation (15) would provide an exact special solution to equation (3) for $\phi$.

There has, as yet, been no specific mention of boundary or initial conditions except to assume that supercritical conditions have somehow given rise to inhomogeneous thermal runaway. Although initial conditions suitable for solving (15) and (16) could always be manufactured by defining, say,

$$
\phi(r, 0)=-\ln t_{I}+g\left(\frac{r-r_{I}}{t_{I}^{\frac{1}{l}}}\right),
$$

it remains to be seen whether or not solutions of the form of equation (15) can have genuine physical relevance, either asymptotically or exactly. In the context of a self-induced supercritical thermal-runaway process two general conditions would need to be met by the function g. First, since $\phi$ is a smooth continuous function of $r$ at all times before the induction time $t_{l}$, it follows that $g$ must be a non-singular function of $s$. Secondly, as the induction time is approached the behaviour of $\phi$ in cooler regions (left behind by the most strongly reacting region as it shrinks towards $r_{I}$ ) is unremarakable. It must therefore be possible to match the Hot-Spot solution (15) with a Taylorseries solution for $\phi$ of the form

$$
\phi \sim \phi\left(r, t_{I}\right)-\left(t_{I}-t\right) \phi_{t}\left(r, t_{I}\right)+\frac{1}{2}\left(t_{I}-t\right)^{2} \phi_{t t}\left(r, t_{I}\right)
$$

as $t \rightarrow t_{\mathrm{I}}$. Each of the functions $\phi\left(r, t_{I}\right), \phi_{\mathrm{t}}\left(r, t_{\mathrm{I}}\right)$, etc. is a well-defined function of $r$, singular only at the induction position $r=r_{I}$, with $\phi_{t}\left(r, t_{I}\right)$, $\phi_{t t}\left(r, t_{\mathrm{I}}\right)$, etc. obtainable from $\phi\left(r, t_{\mathrm{I}}\right)$ by successive time differentiation of equation (3).

It should be noted that this analysis is concerned specifically with spatially varying thermal runaway so that the trivial solution $g=0$ is excluded from 
consideration. This case would represent a completely uniform spatial distribution of tempterature, and has been well investigated by Kassoy (9).

There are two possible forms of asymptotic behaviour for $g$ as $|s|$ grows large:

$$
\left.\begin{array}{l}
g=-\ln s^{2}+A-\frac{2-2 n_{I}+e^{A}}{s^{2}}\left(1-\frac{6-2 n_{I}+e^{A}}{2 s^{2}}\right)+O\left(s^{-6}\right), \\
g=-B^{2} \frac{e^{\frac{1}{x^{2}}}}{|s|^{1+n_{1}}}\left[1+\frac{2\left(1+n_{I}\right)}{s^{2}}\left(1+\frac{2\left(3+n_{I}\right)}{s^{2}}\right)\right]+O\left(\frac{e^{\frac{1 s^{2}}{2}}}{|s|^{7+n_{\mathrm{I}}}}\right)
\end{array}\right\}
$$

When $B$ is non-zero, the latter asymptotic form shows $g$ approaching $-\infty$ at an extremely rapid rate as $|s|$ grows large. With $r$ fixed and not equal to $r_{I}$, the solution for $\phi$ expressed in terms of $r$ and $t$ would also then decrease rapidly towards $-\infty$ as $t$ tends to $t_{r}$. No matching is then possible with the asymptotic form (18) which requires $\phi$ to remain finite in this limit and so, apart from the trivial case $(B=0)$, this form of behaviour cannot be considered to have any physical relevance. The first of the asymptotic forms (19) matches perfectly with the asymptotic form (18) provided that, as $r$ tends to $r_{1}$,

$$
\left.\begin{array}{l}
\phi\left(r, t_{I}\right) \sim-\ln \left(r-r_{I}\right)^{2}+A, \\
\phi_{t}\left(r, t_{I}\right) \sim \frac{2-2 n_{I}+e^{A}}{\left(r-r_{I}\right)^{2}}, \\
\phi_{t t}\left(r, t_{I}\right) \sim \frac{6-2 n_{I}+e^{A}}{\left(r-r_{I}\right)^{2}} \phi_{t}\left(r, t_{I}\right),
\end{array}\right\}
$$

and so on.

It now needs to be seen whether or not any suitably behaved solutions exist. The evidence of a numerical investigation towards this end indicates that no solution of equation (16) can be found to meet all the conditions necessary for physical meaningfulness in the present context. In order to reach this conclusion two families of numerical solutions of (16) were examined: first, those continuous solutions which satisfy the symmetric condition $\dot{g}(0)=0$; and secondly, those solutions which adopt the logarithmic asymptotic behaviour (19), as $|s| \rightarrow \infty$.

Higher derivatives of equation (16) were used as the basis of the numerical scheme: given $g(s)$ and $\dot{g}(s)$, derivatives of $g$ up to $d^{6} g / d s^{6}$ were evaluated directly and used to step the values of $g$ and $\dot{g}$ backwards or forwards using a Taylor-series expression. Backward differencing, which might have introduced stiffness (and hence numerical instability) into the method was thus avoided, and step sizes were limited so as to maintain a high degree of accuracy. This was checked by examining the convergence of 
the scheme with reductions in step sizes. The results given below were all calculated to considerably better than graphical accuracy.

Non-singular solutions in the cylindrically and spherically symmetric cases ( $n_{I}$ equal to 1 or 2 ) must always satisfy the symmetric condition $\dot{g}(0)=0$. In the case of local variation in only one Cartesian dimension $\left(n_{I}=0\right)$ it seems reasonable to expect that, if any suitable solution is to exist, it should be possible to find at least one which satisfies the symmetric condition. For this reason, solutions of equation (16) were obtained by 'shooting' from $s=0$, with $\dot{g}(0)=0$ and with $g(0)$ taking selected values. The advantage of using the shooting method is that every case is, effectively, an initial-value problem with a unique solution. There is not, therefore, the possiblity that each family of solutions obtained might represent only one branch of the general family of solutions.

The solutions sketched in Fig. 2, as examples, all show signs of $|g|$ growing rapidly in the exponential manner, rather than growing gently as it would do in satisfying the logarithmic asymptotic form. Indeed by proceeding to large enough values of $s$, the value of the matching constant $B$ of the asymptotic form (19) ${ }_{2}$ could be determined in every case. Figure 3 shows the resulting variation found for $B$ as a function of $g(0)$, with the arbitrary convention that $B$ is given the same sign as $g(0)$. Interestingly, with this sign convention, $B$ appears to be a smooth function of $g(0)$ in the neighbourhood of the origin $g(0)=0$.

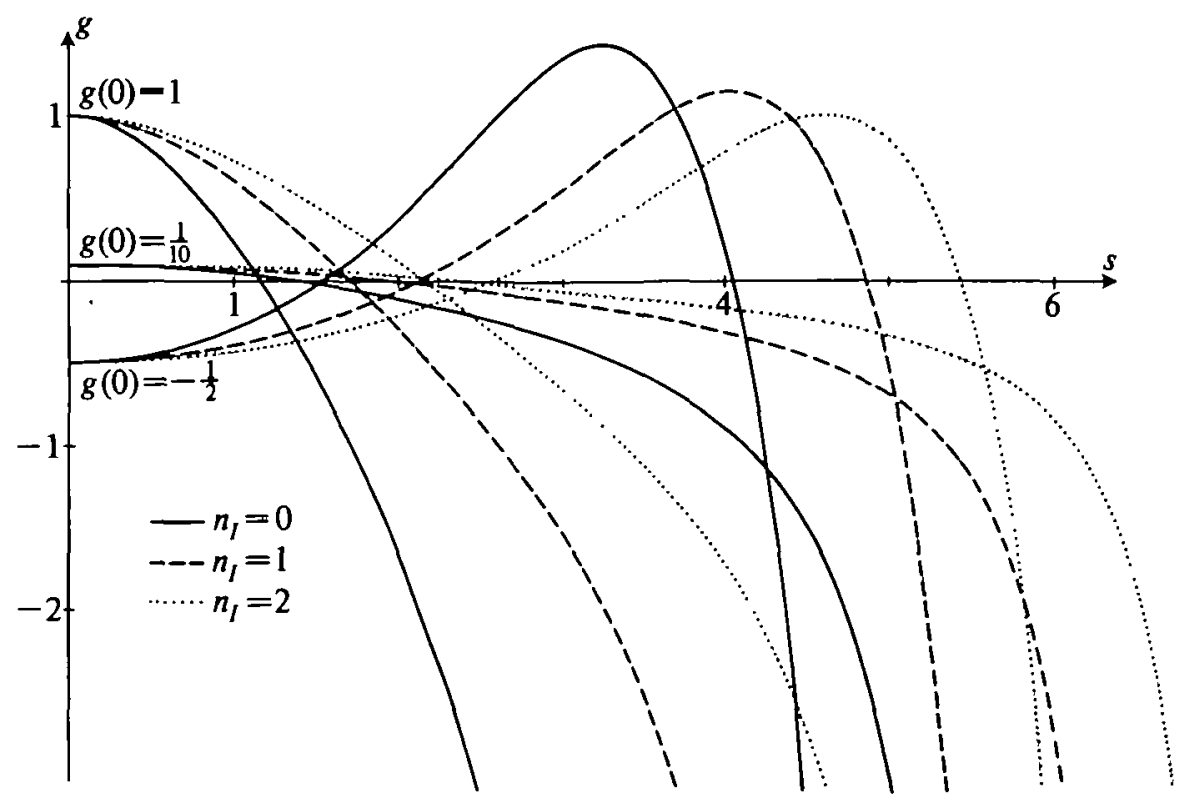

FIG. 2. Numerical solutions for $\mathrm{g}$ satisfying the symmetric condition $\dot{\mathrm{g}}(0)=0$ 


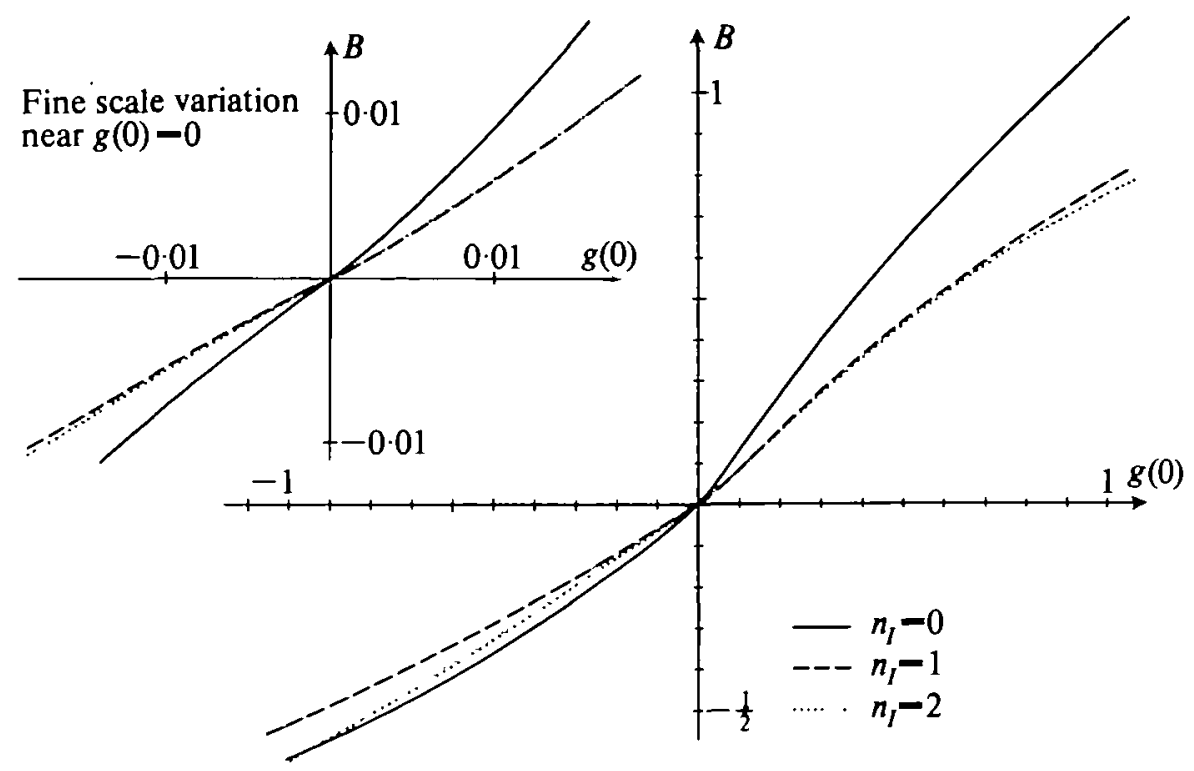

Fio. 3. Exponential matching constant $B$ as a function of $g(0)$ with $\dot{g}(0)=0$

If $\mathbf{g}$ were to adopt its logarithmic asymptotic form for some non-trivial value $g_{I}$ of $g(0)$, then the corresponding value of $B$ would be zero: and, if the family of functions $g$ were to vary continuously with $g(0)$, then one would expect the computed values of $B$ to tend towards zero as $g(0)$ tended to $g_{I}$. In Fig. 3 the only stage at which $B$ can be seen to tend to zero is the trivial limit $g(0)=0$, which leads to the trivial solution $g \equiv 0$. Hence there is nothing in these curves to indicate that a value of $g(0)$ for which the solution would adopt the logarithmic asymptotic form could be found.

One is led to conclude that no symmetric solutions suitable for describing inhomogeneous thermal runaway can be found. In the cases of cylindrical and spherical symmetry it can be deduced that any solutions which do adopt the logarithmic asymptotic form must be singular at $s=0$. In the case of local variation in only one Cartesian dimension there still remains the possibility that, with $\dot{g}(0)$ non-zero, $g$ might be non-singular, non-symmetric and still able to adopt the logarithmic asymptotic form both as $s \rightarrow+\infty$ and $s \rightarrow-\infty$.

Figure 4 illustrates the behaviour of solutions which satisfy the logarithmic form $(19)_{1}$ for large positive values of $s$. These were obtained using the shooting method by starting at sufficiently large values of $s$ (with $g(s)$ and $\dot{\mathrm{g}}(s)$ determined initially by the asymptotic form $\left.(19)_{1}\right)$ and shooting backwards. As expected, the cylindrically and spherically symmetric solutions are singular at $s=0$. In the one-dimensional case $\left(n_{I}=0\right)$, with $g$ behaving logarithmically as $s \rightarrow+\infty$, the solutions obtained all have $\dot{g}(0)$ negative and 


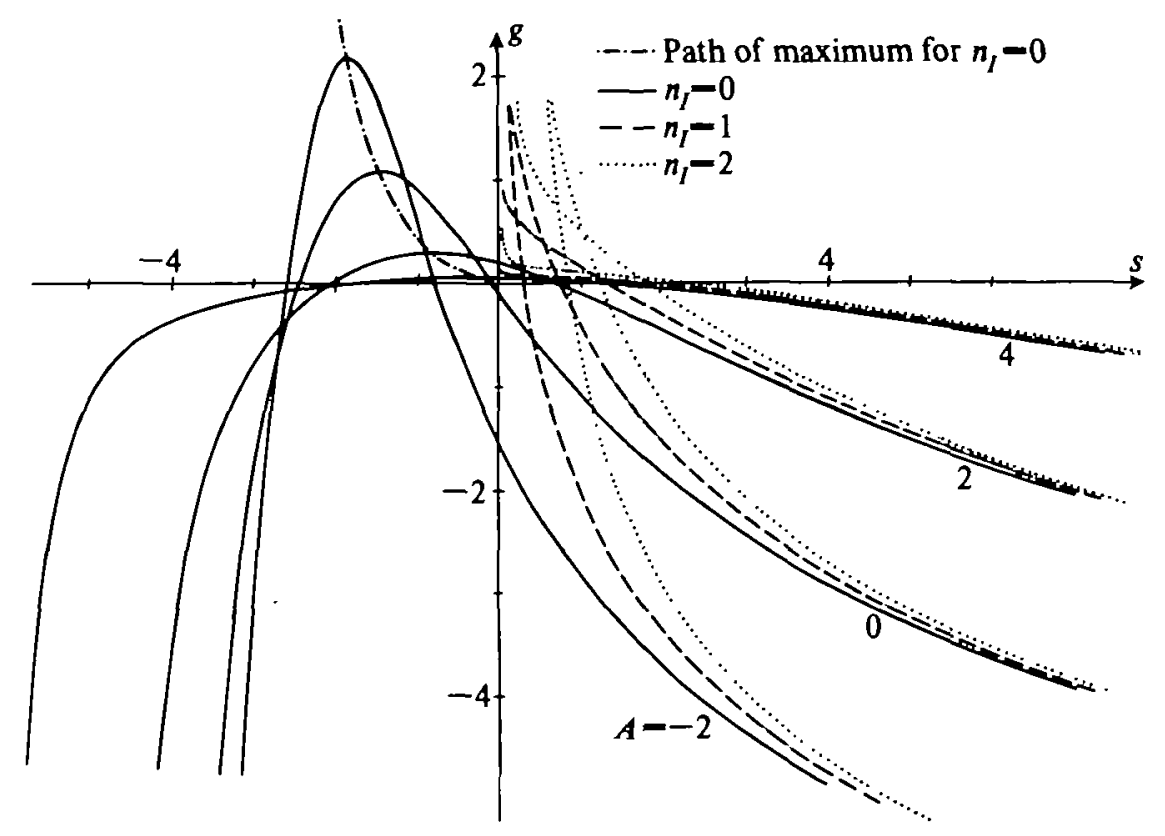

Fro. 4. Numerical solutions for $\mathrm{g}$ behaving logarithmically for large values of $s$

$g$ possessing a positive maximum value for a negative value of $s$. The dot-dashed line of Fig. 4 shows the path of the position of this maximum value. As $s$ grows large and negative the solutions sketched all clearly show signs of adopting the exponential asymptotic form $(19)_{2}$ and hence still failing to match with any asymptotic behaviour of $g$ of the form (18).

Simply changing the sign of $s$ covers the case of solutions behaving logarithmically as $s \rightarrow-\infty$. The question of finding a solution which behaves logarithmically both as $s \rightarrow+\infty$ and $s \rightarrow-\infty$ must therefore depend on being able to find two solutions possessing the logarithmic asymptotic form as $s \rightarrow+\infty$ with the same value of $g$ at $s=0$ but with slopes of opposite sign. Thus at least one solution must be found with positive slope at $s=0$. And, since $g \rightarrow-\infty$ as $s \rightarrow+\infty$, the intermediate value theorem shows that this solution must possess a maximum value for a positive value of $s$. In all cases only one maximum value was encountered, and for the range of curves reliably represented in Fig. 4 these maxima clearly occur only for negative values of $s$. Figure 5 shows the path of the position of the maximum near the origin for large values of the matching constant $A$. As $A$ grows the maximum position moves towards the origin, but there is no indication that the maximum position might swap over to a positive value of $s$ for any value of $\boldsymbol{A}$. Indeed, if this were to happen continuously the intermediate value theorem would require at least one solution to have its maximum at $s=0$. 


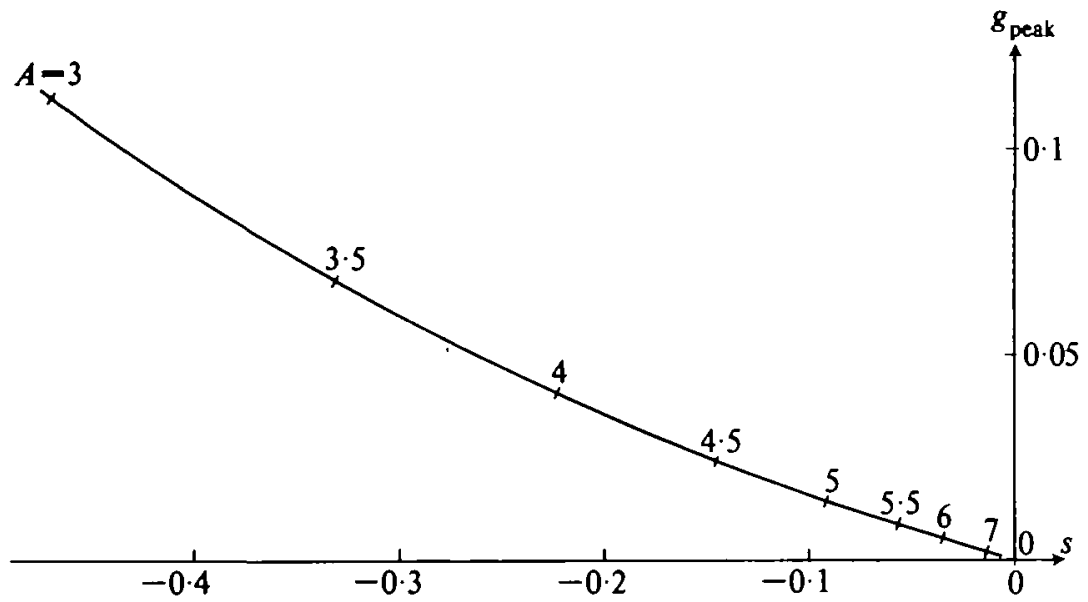

Fig. 5. Path of the maximum value of $g$ as $A$ increases (one-dimensional case, $n_{I}=0$ )

This solution would be symmetric $(\dot{g}(0)=0)$ and it has already been seen that such solutions do not behave logarithmically as $|s| \rightarrow \infty$. Evidently no non-singular solution of (16) which does not adopt the exponential rather than logarithmic asymptotic form at any stage can be found to exist. Hence, according to the criteria identified between equations (17) and (20), there appears to be no physically meaningful Hot-Spot solution.

Practical attempts presented by other authors $(5,6,10)$ to use the Hot-Spot variable grouping to model thermal runaway all concentrate on the case in which $n_{I}=0$, with large values of $A$ in the logarithmic asymptotic form (19) ${ }_{1}$. As can be seen from Fig. 4, with the curve for $A=4$ being small, it is possible in such cases to miss the essential point that $\dot{g}(0)$ is non-zero and so to assume, by symmetry, that matching with the expansion (18) would be possible for both positive and negative values of $s$. One is subtly encouraged in this assumption by the seductive neatness of the matching process which would occur with the logarithmic asymptotic behaviour of $g$.

The spatial discretizations used by $(5,6)$ in numerically solving a version of equation (3) are acknowledged to become inadequate as localized thermal runaway sets in. These solutions therefore fail to be accurate enough at a critical stage which might otherwise have been useful for testing the assumptions of the Hot-Spot analysis. One test, for instance, could have been made by comparing the computed values of the second derivative $\phi_{r r}$ at the peak value of $\phi$ with those predicted by the asymptotic form (15). The definition (14) of the Hot-Spot variable grouping shows that, if the Hot-Spot expansion were to be valid, one should expect $\left|\phi_{r r}\right|_{\text {peak }}$ to grow in proportion to $\left(t_{I}-t\right)^{-1}$ as $t$ tends to $t_{I}$. Numerically more accurate solutions of equation (3) 


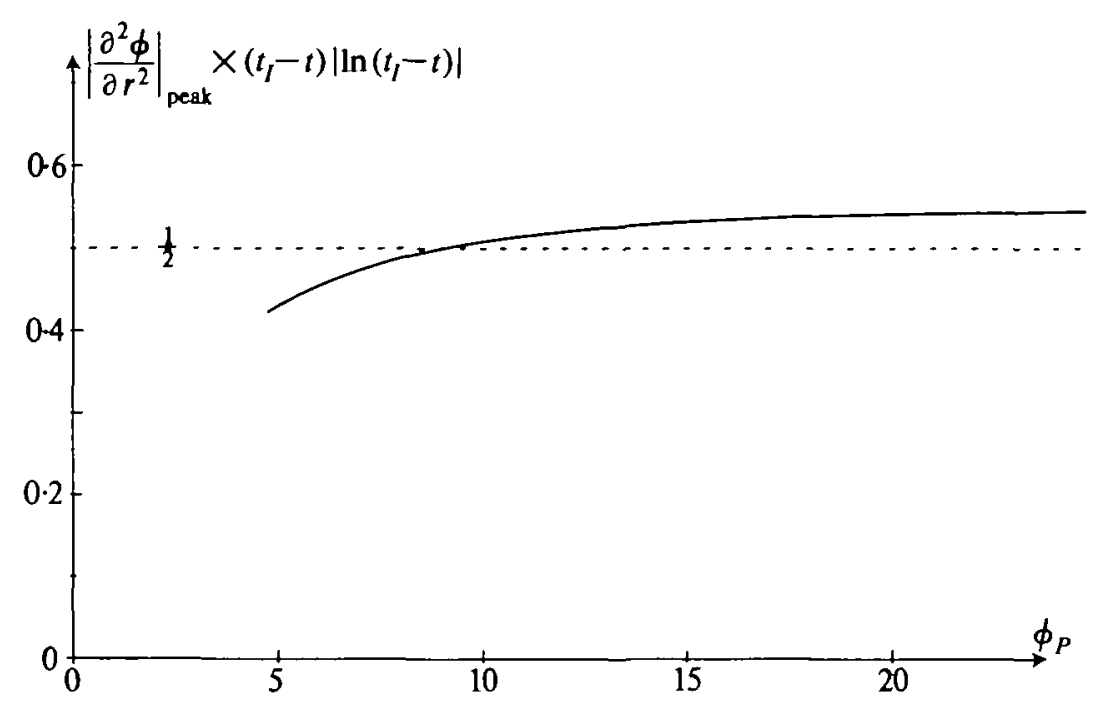

FIG. 6. A comparison of $\left|\partial^{2} \phi / \partial r^{2}\right|_{\text {peak }} \times\left(t_{I}-t\right)\left|\ln \left(t_{I}-t\right)\right|$ with the value $\frac{1}{2}$

were obtained as described below. They, in fact, showed $\left|\phi_{r}\right|_{\text {peak }}$ tending to grow in proportion to $\left[\left(t_{I}-t\right)\left|\ln \left(t_{I}-t\right)\right|\right]^{-1}$ as illustrated in Fig. 6. This sort of behaviour is entirely consistent with the analysis based on the IgnitionKernel variable grouping (see equations (46)).

Equation (3) was solved numerically by following the time development of temperature at fixed, uniformly-distributed, spatial points. To do this $\phi_{t}$, and successively $\phi_{t t}$ and $\phi_{t t}$, were calculated at each point using equation (3) and time derivatives of equation (3), with eighth-order polynomial-fitting formulae (centrally based over nine points) used for estimating the relevant spatial derivatives. Using one order of backward differencing for increased accuracy, time-stepping was then performed by means of a Taylor series in $\phi$. Step sizes were limited so as to maintain a given degree of accuracy, as well as to prevent numerical instability. 'Ghost' points were used to impose boundary conditions, such as condition $(50)_{1}$ below.

As thermal runaway proceeds the concentration of the growth of $\phi$ about a maximum value leads to reduced accuracy in the estimates of the spatial derivatives in this region. A formula, based on the eighth-order coefficient in the polynomial fitting, was used to estimate this loss of accuracy at every stage. As soon as it exceeded a given small level, mid-point interpolation was used to add points to the discretization, thereby improving the resolution before substantial numerical errors could be accrued. The problem of progressively adding unlimited numbers of points as the temperature continues to rise was overcome by ignoring temperatures colder than the peak temperature by a given 'cold-boundary' level at which the temperature was allowed to grow linearly. Results proved to remain accurate for quite 
moderate choices of cold-boundary level. The balance of accuracy in timestepping, level of point addition and cold-boundary level was optimized to maintain a high degree of accuracy in all cases calculated.

\section{Ignition-Kernel coordinate-perturbation solution}

\section{a. Derivation}

With $\sigma$ defined as $\xi$, or $-\ln \left(t_{I}-t\right)$, the variable grouping $\eta$ of equation $(6)_{2}$ takes on a form of the Ignition-Kernel variable grouping:

$$
\eta=\frac{r-r_{I}}{\left[\xi e^{-\xi}\right]^{\xi}}=\frac{r-r_{I}}{\left[\left(t_{I}-t\right) \mid \ln \left(t_{I}-t\right)\right]^{\beta}} .
$$

Because of the small difference between this definition and the more general one of equation (2) the symbol $\eta$, rather than $\rho$, will be retained to distinguish this particular variable grouping. Its relationship to the more general form will be considered in section 5 . Since $\phi$ grows primarily as $-\ln \left(t_{I}-t\right)$, it is convenient to make a transformation to $W$ such that

$$
W=\frac{e^{-\phi}}{t_{I}-t}=e^{\epsilon-\phi} .
$$

One should then expect $W$ to behave in a non-trivial order-one fashion as a function of $\eta$ and $\xi$. It satisfies the partial differential equation

$$
\begin{aligned}
W_{\xi}+\frac{1}{2} \eta W_{\eta}+\lambda\left(r_{I}+\right. & {\left.\left[\xi e^{-\xi}\right]^{\frac{1}{2}} \eta, t_{I}-e^{-\xi}\right) } \\
& =W+\xi^{-1}\left[\frac{\eta}{2} W_{\eta}+W_{m}+\frac{n\left[\xi e^{-\xi}\right]^{\frac{1}{2}}}{r_{I}+\left[\xi e^{-\xi}\right]^{\frac{1}{2} \eta}} W_{\eta}-\frac{W_{\eta}^{2}}{W}\right]
\end{aligned}
$$

in which, conveniently, only one nonlinear term appears. As far as possible it is proposed to solve for $W$ as a coordinate-perturbation expansion of the form

$$
W \sim W_{1}(\eta)+\dot{\gamma}_{2}(\xi) W_{2}(\eta)+\ldots+\gamma_{k}(\xi) W_{k}(\eta)
$$

as $\xi \rightarrow \infty$ with $\eta$ fixed, using only the assumption that for all orders $k, W_{k}(\eta)$ is a non-singular non-trivial function of $\eta$.

This immediately gives, to first order,

whence

$$
\frac{1}{2} \eta \dot{W}_{1}+1=W_{1},
$$

$$
W_{1}=1+c_{1} \eta^{2} / 4 \text {. }
$$

In the absence of boundary conditions the integration constant $c_{1}$ cannot be determined at this stage. But this need not hinder an examination of higher orders in the expansion with $c_{1}$ being left arbitrary for the time being. Concerning the second order, all that can be said initially about $\gamma_{2}$ is that it 
must be in the range

$$
O\left(\xi^{-1}\right) \leqslant \gamma_{2}(\xi) \ll 1
$$

Since the possibility of having

$$
\gamma_{2}(\xi) \gg \xi^{-1}
$$

cannot be ruled out it will be assumed, for the present, that this is in fact the case. The second-order terms must then satisfy

$$
\frac{\eta}{2} \dot{W}_{2}=\left(1+\left[\frac{\dot{\gamma}_{2}}{\gamma_{2}}\right]^{*}\right) W_{2}
$$

where the notation [ ] $]^{*}$ has the same meaning as in equation (10). The case of $\left[\dot{\gamma}_{2} / \gamma_{2}\right]^{*}$ being infinite can be discounted as this would lead to a trivial result. It can then be shown that condition (26) can only be satisfied if $\left[\dot{\gamma}_{2} / \gamma_{2}\right]^{*}$ is zero. Thus (28) gives

$$
W_{2}=c_{2} \eta^{2} / 4
$$

Proceeding to third order and assuming, conditionally, that

$$
\xi^{-1} \ll \gamma_{3}^{\prime}(\xi) \ll \gamma_{2}(\xi)
$$

(note that the prime denotes the conditional nature of this assumption), gives

whence

$$
\frac{1}{2} \eta \dot{W}_{3}^{\prime}=W_{3}^{\prime},
$$

$$
W_{3}^{\prime}=c_{3}^{\prime} \eta^{2} / 4
$$

where, to satisfy condition (26), the conditions that $\left[\dot{\gamma}_{3}^{\prime} / \gamma_{3}^{\prime}\right]^{*}$ and $\left[\dot{\gamma}_{2} / \gamma_{3}^{\prime}\right]^{*}$ must be zero, have been used. Since $W_{3}^{\prime}$ is identical in form to $W_{2}$, it follows that $\gamma_{2}$ could be redefined in the manner

$$
\gamma_{2}=\gamma_{2}^{\prime}+\frac{c_{3}^{\prime}}{c_{2}} \gamma_{3}^{\prime} \text {. }
$$

By repeating the process, if necessary, any term whose order is in the range (30) can be made redundant. Assuming that such a judicious choice of $\gamma_{2}$ has been made, one is free to define

$$
\gamma_{3}=\xi^{-1} \text {. }
$$

Again it follows from condition (26) that $\left[\xi \dot{\gamma}_{2}\right]^{*}$ must be zero. Only the first-order term in the expansion then contributes to the equation satisfied by $W_{3}$ :

$$
\begin{aligned}
\frac{\eta}{2} \dot{W}_{3}-W_{3} & =\ddot{W}_{1}+\left(\frac{n_{1}}{\eta}+\frac{\eta}{2}\right) \dot{W}_{1}-\frac{\dot{W}_{1}^{2}}{W_{1}} \\
& =\frac{c_{1}}{2}+\left(\frac{n_{1}}{\eta}+\frac{\eta}{2}\right) c_{1} \frac{\eta}{2}-c_{1}^{2} \frac{\eta^{2} / 4}{1+c_{1} \eta^{2} / 4}
\end{aligned}
$$


giving

$$
\begin{aligned}
W_{3}= & 2 \eta^{2} \int \frac{c_{1}}{\eta^{3}}\left\{\frac{1+n_{I}}{2}+c_{1} \frac{\eta^{4} / 16}{1+c_{1} \eta^{2} / 4}+\left(1-c_{1}\right) \frac{\eta^{2} / 4}{1+c_{1} \eta^{2} / 4}\right\} d \eta \\
= & c_{1} \frac{\eta^{2}}{4}\left[c_{3}+\ln \left(1+c_{1} \eta^{2} / 4\right)\right]-c_{1} \frac{1+n_{I}}{2}+ \\
& +\left(1-c_{1}\right) c_{1} \frac{\eta^{2}}{4} \ln \left(\frac{\eta^{2}}{1+c_{1} \eta^{2} / 4}\right) .
\end{aligned}
$$

Since, by hypothesis, the thermal runaway is spatially varying, $c_{1}$ cannot have the value zero. Thus, unless $c_{1}$ has the value unity, the final term in the expression for $W_{3}$ will have a singular second derivative. As argued following equation (11) this would contribute a singular effect to the term of order $\xi^{-2}$ via equation (23). This is clearly unacceptable since there are no singularities in the development of $\phi$ before the induction time is reached. It follows that

$$
c_{1}=1 \text {. }
$$

It may be noted that the term thus eliminated from the solution for $W_{3}$ arises from a pole of order $\eta^{-1}$ under the integral sign of the integral expression (34). This, in turn, arises from the term of order $\eta^{2}$ in a power-series expansion of the right-hand side of the differential equation. Condition (35) could thus have been deduced directly from the differential equation for $W_{3}$ by eliminating the order $\eta^{2}$ term. It can also be seen that this elimination can only be carried out, non-trivially, because of the nonlinear term $W_{\eta}^{2} / W$ of equation (23), whose effect is to introduce a quadratic dependence on $c_{1}$ into the differential equation for $W_{3}$.

The first-order term in the expansion is thus fully determined by applying a smoothness condition to the term of order $\xi^{-1}$. No additional boundary condition has been necessary. In this way higher-order effects can be seen to bring the nonlinearity of the problem into operation in enforcing a uniquely defined form for the growth of $\phi$ in the Ignition Kernel, at least to first order.

Proceeding further with the analysis in the same way, the following fourth- and fifth-order results can be obtained:

with

$$
\gamma_{4}=\gamma_{2} / \xi
$$

$$
\begin{aligned}
\frac{\eta}{2} \dot{W}_{4}-W_{4} & =\ddot{W}_{2}+\left(\frac{n_{I}}{\eta}+\frac{\eta}{2}\right) \dot{W}_{2}-\left[\frac{\xi \dot{\gamma}_{2}}{\gamma_{2}}\right]^{*} W_{2}-\frac{2 \dot{W}_{1} \dot{W}_{2}}{W_{1}}+\frac{\dot{W}_{1}^{2} W_{2}}{W_{1}^{2}} \\
& =c_{2}\left\{\frac{1+n_{I}}{2}-\left(1+\left[\frac{\xi \dot{\gamma}_{2}}{\gamma_{2}}\right]^{*}\right) \frac{\eta^{2}}{4}+\frac{\eta^{4}}{16} \frac{3+2 \eta^{2} / 4}{\left(1+\eta^{2} / 4\right)^{2}}\right\}
\end{aligned}
$$

and

$$
\gamma_{5}=\xi^{-2}
$$


with

$$
\begin{aligned}
\frac{\eta}{2} \dot{W}_{5}-W_{5}= & W_{3}-\left[\xi^{2}\left(\dot{\gamma}_{2}-\left[\frac{\xi \dot{\gamma}_{2}}{\gamma_{2}}\right]^{*} \frac{\gamma_{2}}{\xi}\right)\right]^{*} W_{2}+\ddot{W}_{3}+ \\
& +\left(\frac{n_{I}}{\eta}+\frac{\eta}{2}\right) \dot{W}_{3}-\frac{2 \dot{W}_{1} \dot{W}_{3}}{W_{1}}+\frac{\dot{W}_{1}^{2} W_{3}}{W_{1}^{2}} \\
= & \frac{1+n_{1}}{2}\left[c_{3}-1+\ln \left(1+\eta^{2} / 4\right)-\frac{\eta^{2} / 4}{\left(1+\eta^{2} / 4\right)^{2}}\right]+ \\
& +\left(\frac{5+n_{I}}{2}-\left[\xi^{2}\left(\dot{\gamma}_{2}-\left[\frac{\xi \dot{\gamma}_{2}}{\gamma_{2}}\right]^{*} \frac{\gamma_{2}}{\xi}\right)\right]^{*} c_{2}\right) \frac{\eta^{2}}{4}+ \\
& +\frac{\eta^{4}}{16} \frac{3+2 \eta^{2} / 4}{\left(1+\eta^{2} / 4\right)^{2}}\left[c_{3}+\ln \left(1+\eta^{2} / 4\right)\right] \\
& -\frac{\eta^{4}}{16} \frac{2-\eta^{2} / 4}{\left(1+\eta^{2} / 4\right)^{2}}-\frac{5+n_{1}}{2} \frac{\eta^{4} / 16}{1+\eta^{2} / 4} .
\end{aligned}
$$

Eliminating the coefficients of $\eta^{2}$ from these equations, as required, leads to the conditions

$$
\left.\begin{array}{l}
\left(1+\left[\frac{\xi \dot{\gamma}_{2}}{\gamma_{2}}\right]^{*}\right) c_{2}=0, \\
{\left[\xi^{2}\left(\dot{\gamma}_{2}-\left[\frac{\xi \dot{\gamma}_{2}}{\gamma_{2}}\right]^{*} \frac{\gamma_{2}}{\xi}\right)\right]^{*} c_{2}=\frac{5+n_{1}}{2} .}
\end{array}\right\}
$$

The latter condition rules out the possible trivial solution for $c_{2}$ in the former condition which therefore shows that $\left[\xi \dot{\gamma}_{2} / \gamma_{2}\right]^{*}$ must have the value -1 . Using the degree of freedom inherent in the definition of $\gamma_{2}$ to define a value for $c_{2}$, the latter condition now becomes, for instance,

$$
\left.\begin{array}{l}
{\left[\xi^{2}\left(\dot{\gamma}_{2}+\frac{\gamma_{2}}{\xi}\right)\right]^{*}=1,} \\
c_{2}=\frac{1}{2}\left(5+n_{1}\right) .
\end{array}\right\}
$$

As a result it can be deduced that $\gamma_{2}$ has the form

where

$$
\gamma_{2}=\frac{\ln \xi}{\xi}+\mu,
$$

$$
\mu(\xi)=o\left(\frac{\ln \xi}{\xi}\right) .
$$

This result for $\gamma_{2}$ can easily be refined a step further by proceeding to sixth order. For this it can be shown that one may take

$$
\gamma_{6}=\frac{\gamma_{2}^{2}}{\xi}=\xi^{-1}\left(\frac{\ln \xi}{\xi}+\mu\right)^{2},
$$


giving

$$
\begin{aligned}
\frac{\eta}{2} \dot{W}_{6}-W_{6}= & -\left[\frac{\xi^{3}}{\ln ^{2} \xi}\left(\dot{\mu}+\frac{\mu}{\xi}\right)\right]^{*} W_{2}-\frac{\dot{W}_{2}^{2}}{W_{1}}+\frac{2 \dot{W}_{1} \dot{W}_{2} W_{2}}{W_{1}^{2}}-\frac{\dot{W}_{1}^{2} W_{2}^{2}}{W_{1}^{3}} \\
= & -\left(\left[\frac{\xi^{3}}{\ln ^{2} \xi}\left(\dot{\mu}+\frac{\mu}{\xi}\right)\right]^{*}+\frac{5+n_{I}}{2} \frac{1}{1+\frac{1}{4} \eta^{2}}\right) \frac{5+n_{1}}{2} \frac{\eta^{2}}{4}+ \\
& +\left(\frac{5+n_{I}}{2}\right)^{2} \frac{\eta^{4}}{16} \frac{2+\frac{1}{4} \eta^{2}}{1+\frac{1}{4} \eta^{2}},
\end{aligned}
$$

which leads to the condition

$$
\left[\frac{\xi^{3}}{\ln ^{2} \xi}\left(\dot{\mu}+\frac{\mu}{\xi}\right)\right]^{*}+\frac{5+n_{I}}{2}=0,
$$

from which it can be shown that, as $\xi$ tends to infinity,

$$
\mu \sim \frac{5+n_{I}}{2} \frac{\ln ^{2} \xi}{\xi^{2}} .
$$

Since $\mu$ is asymptotically smaller than $\xi^{-1}$ the result of considering six orders in the expansion has thus been to produce an expansion for $W$ accurate to the third order, namely,

$$
\begin{aligned}
W= & 1+\frac{\eta^{2}}{4}+\frac{\ln \xi}{\xi} \frac{5+n_{I}}{2} \frac{\eta^{2}}{4}+ \\
& +\xi^{-1}\left\{\frac{\eta^{2}}{4}\left[c_{3}+\ln \left(1+\eta^{2} / 4\right)\right]-\frac{1+n_{I}}{2}\right\}+O\left(\frac{\ln ^{2} \xi}{\xi^{2}}\right) .
\end{aligned}
$$

The pattern may be continued to yet higher orders, with progressive refinements of the gauge function $\gamma_{2}$ emerging in the process. In fact, beyond the third order, every application of the condition of smoothness involves the limiting value of an expression containing the derivative of $\gamma_{2}$ with respect to $\xi$ and thereby serves only to refine the asymptotic estimate of $\gamma_{2}$. No further conditions, such as equation (35), emerge to determine the third-order constant of integration $c_{3}$, or any other higher-order constants, which can thus be seen to serve as parametric constants of the expansion, acquiring values appropriate to the particular thermal-runaway situation.

\section{b. Properties}

Using the transformation (22) the Ignition-Kernel expansion for $\phi$, to third order, can be written as

$$
\begin{aligned}
\phi= & \xi-\ln \left(1+\frac{1}{4} \eta^{2}\right)-\frac{\ln \xi}{\xi} \frac{5+n_{I}}{2} \frac{\frac{1}{4} \eta^{2}}{1+\frac{1}{4} \eta^{2}}+ \\
& +\xi^{-1}\left\{\frac{1+n_{I}}{2}+\frac{\frac{1}{4} \eta^{2}}{1+\frac{1}{4} \eta^{2}}\left[\alpha_{I}-\ln \left(1+\frac{1}{4} \eta^{2}\right)\right]\right\}+O\left(\frac{\ln ^{2} \xi}{\xi^{2}}\right),
\end{aligned}
$$


where

$$
\alpha_{I}=-\frac{1}{2}\left(1+n_{I}\right)-c_{3} .
$$

It is a remarkable consequence of the nonlinearity of the problem that, being uniquely determined, the first two orders in the Ignition-Kernel expansion are independent of the conditions leading up to thermal runaway. Of course, the very existence of thermal runaway, and the vital induction parameters $r_{I}$ and $t_{\mathrm{I}}$, are far from being independent.

A further notable aspect of the Ignition-Kernel expansion is its relative independence of the three different possible topologies of the thermalrunaway region, denoted by the values of $n_{I}$ defined in equation (10). Whether the thermal runaway is localized about a point, a line or a surface, the shape of the temperature profile is identical to first order. The reason for this lies, most directly, with the nonlinear term of equation (34). Just as the only nonlinear term, $W_{\eta}^{2} / W$, of equation (23) arises solely from the transformation of the term consisting of the second spatial derivative of $\phi$ (which is independent of $n$ ) in equation (3), this term is also independent of $n$. This in turn is a consequence of the exponential nature of the nonlinearity of equation (3), which is encapsulated in the transformation (22).

In fact the relative importance of conduction, through which the effect of the topology is felt, can be seen to decrease to asymptotically small values as $\xi$ increases. Using (45) to evaluate the conduction- and reaction-rate terms of equation (3) in the Ignition-Kernel region gives, to first order,

$$
\left.\begin{array}{l}
\phi_{r r}+\frac{n}{r} \phi_{r} \sim-\frac{e^{\xi}}{\xi} \frac{\left(n_{\mathrm{r}}+1\right)+\left(n_{\mathrm{I}}-1\right) \eta^{2} / 4}{2\left(1+\frac{1}{4} \eta^{2}\right)^{2}}, \\
\lambda(r, t) e^{\phi} \sim \frac{e^{\xi}}{1+\frac{1}{4} \eta^{2}},
\end{array}\right\}
$$

respectively. Thus the ratio of the conduction- to the reaction-rate terms can be seen to fall off like $\xi^{-1}$ as the Ignition Kernel develops. This behaviour is consistent with the numerical findings already discussed and presented in Fig. 6.

The reduction in the importance of conductive effects reflects the role of the logarithmic factor in the definition of $\eta$. Relatively speaking, the temperature profile spreads out spatially or, rather, the spatial aspects are not as rapidly concentrated as the temporal aspects.

\section{Generalization of the solution}

The more general Ignition-Kernel variable grouping $\rho(r, t ; \alpha)$, presented in equation (2), can be written in the form

$$
\rho=\frac{\left(r-r_{I}\right) / e^{\frac{1}{2} \alpha}}{\left[\zeta e^{-\zeta}\right]^{\frac{1}{2}}}=\frac{\eta}{[1+(\alpha / \xi)]^{\frac{1}{2}}},
$$


where $e^{-\zeta}=\left(t_{I}-t\right) / e^{\alpha}$ or $\zeta=\alpha+\xi$. In effect this uses the parameter $\alpha$ to normalize the Ignition-Kernel variable grouping such that when $\zeta$ and $\rho$ are both of order one, $t_{I}-t$ and $\left(r-r_{I}\right)^{2}$ are both of order $e^{\alpha}$. In terms of $\rho$ and $\zeta$ the third-order asymptotic solution (45) for $\phi$ takes on the form

$$
\begin{aligned}
\phi+\alpha= & \frac{\alpha_{I}-\alpha}{\zeta} \frac{\frac{1}{4} \rho^{2}}{1+\frac{1}{4} \rho^{2}}+\zeta-\ln \left(1+\frac{1}{4} \rho^{2}\right)-\frac{\ln \zeta}{\zeta} \frac{5+n_{I}}{2} \frac{\frac{1}{4} \rho^{2}}{1+\frac{1}{4} \rho^{2}}+ \\
& +\zeta^{-1}\left[\frac{1+n_{I}}{2}-\frac{\frac{1}{4} \rho^{2}}{1+\frac{1}{4} \rho^{2}} \ln \left(1+\frac{1}{4} \rho^{2}\right)\right]+O\left(\frac{\ln ^{2} \zeta}{\zeta^{2}}\right)
\end{aligned}
$$

It can be seen from this that a suitable choice for $\alpha$ can be made to remove the constant $\alpha_{I}$ from the expansion for $\phi+\alpha$ to third order, absorbing the influence of $\alpha_{I}$ into the definition of $\rho$. A uniquely defined third-order form for $\phi+\alpha$ in terms of $\rho$ and $\zeta$ can thus be obtained.

An appropriate choice of $\alpha$ is to define $\alpha(\zeta)$ simply as the constant $\alpha_{\text {I }}$, giving

$$
\begin{aligned}
\phi+\alpha_{I}= & \zeta-\ln \left(1+\frac{1}{4} \rho^{2}\right)-\frac{\ln \zeta}{\zeta} \frac{5+n_{I}}{2} \frac{\frac{1}{4} \rho^{2}}{1+\frac{1}{4} \rho^{2}}+ \\
& +\zeta^{-1}\left[\frac{1+n_{I}}{2}-\frac{\frac{1}{4} \rho^{2}}{1+\frac{1}{4} \rho^{2}} \ln \left(1+\frac{1}{4} \rho^{2}\right)\right]+O\left(\frac{\ln \zeta}{\zeta^{2}}\right)
\end{aligned}
$$

where

$$
\rho^{2}=\frac{\left(r-r_{I}\right)^{2} / e^{\alpha_{1}}}{\zeta e^{-\zeta}}, \quad e^{-\zeta}=\frac{t_{I}-t}{e^{\alpha_{I}}}
$$

(Note that if $\alpha(\zeta)$ were chosen to equal $\alpha_{I}-\frac{1}{2}\left(5+n_{I}\right) \ln \zeta$, then the term containing $\ln \zeta$ could also have been eliminated from equation (48).) This asymptotic form indicates that when $\zeta$ is of order one $\phi$ is within order one of $-\alpha_{I}$. At this stage $\phi_{t}, \phi_{r r}$ and $e^{\phi}$ all have similar orders of magnitude, namely $e^{-\alpha_{1}}$. Thus $-\alpha_{I}$ represents a value of $\phi$ near which thermal conductive effects would be of significant importance in hindering the growth of $\phi$. The expansion (49) is not asymptotically valid at this stage, but becomes a more accurate representation as $\phi$ increases. Broadly speaking, $-\alpha_{I}$ represents a threshold value of $\phi$ in relation to which the thermal-runaway process takes off in earnest.

Assuming that $\phi(r, t)$ has order-one initial or boundary conditions, $\alpha_{I}$ can thus be interpreted as some measure of the degree of supercriticality of the problem; for highly supercritical situations $\alpha_{I}$ can be expected to be large. This is strongly borne out by numerical results presented in the next section, where $\alpha_{I}$ is found to increase rapidly as conditions become more supercritical.

The advantage of absorbing $\alpha_{I}$ into the definitions of $\rho$ and $\zeta$ now becomes clear. Since its third-order part is proportional to $\alpha_{1} / \xi$, when $\alpha_{I}$ is large the asymptotic form (45) can only be expected to be accurate for very 
large values of $\xi$ or $\phi$. The result of removing $\alpha_{I}$ from the asymptotic form into the definition of the variable grouping is to produce a more uniformly accurate expansion, regardless of the value of $\alpha_{I}$. Indeed, since $\zeta$ grows primarily like $\phi+\alpha_{I}$ this asymptotic form is likely to be accurate over a wider range of values of $\phi$ for greater values of $\alpha_{\mathrm{I}}$.

A more universal underlying structure in the thermal-runaway process is also revealed: examining the expansion (49) it can be seen that the behaviour of $\phi$ when $\alpha_{I}$ is large is very much like its behaviour when $\phi$ is large and $\alpha_{I}$ is not large. That is, a strongly supercritical thermal runaway is similar to a less strongly supercritical, but more highly-developed, thermal runaway.

\section{Resnlts for a fixed-temperature container}

Thermal runaway in a symmetric container of constant temperature may be modelled using equation (3) with the conditions

$$
\phi(a, t)=0, \quad \lambda(r, t)=1,
$$

where $a$ represents the size of the container, which may be slab-shaped, cylindrical or spherical, for $n$ equal to 0,1 or 2 respectively. This formulation can be identified with the more classical Frank-Kamanetskii formulation (1), in which $\lambda$ is uniformly defined to take the value of the FrankKamanetskii parameter $\delta$ with $\phi(1, t)=0$. The two formulations correspond if $r$ and $t$ are rescaled so that $r^{\prime}=r / \delta^{t}, t^{\prime}=t / \delta$ and if $a$ is defined to take the value $\delta^{\frac{1}{2}}$. If $a$ exceeds a critical value $a_{\mathrm{cs}}$ where

$$
a_{\mathrm{cr}}= \begin{cases}0.937, & n=0, \\ \sqrt{2}, & n=1, \\ 1.823, & n=2,\end{cases}
$$

then no steady-state solution of equation (3) under condition (50) can be found: thermal runaway must ultimately occur.

In the manner described at the end of section 3, the time development of $\phi$, starting with the constant initial value

$$
\phi(r, 0)=0
$$

and keeping condition (50) satisfied, was obtained numerically for each value of $n$. The results for $t_{I}$ (sketched as $1 / t_{I}^{2}$ ) and $\alpha_{I}$ are shown in Figs 7 and 8. As anticipated, $\alpha_{I}$ increases with increasing supercriticality, starting at the values,

$$
\alpha_{I_{\sigma}}= \begin{cases}-2 \cdot 68, & n=0, \\ -1 \cdot 94, & n=1, \\ -1 \cdot 73, & n=2,\end{cases}
$$

for $a$ very close to $a_{\mathrm{cr}}$. 


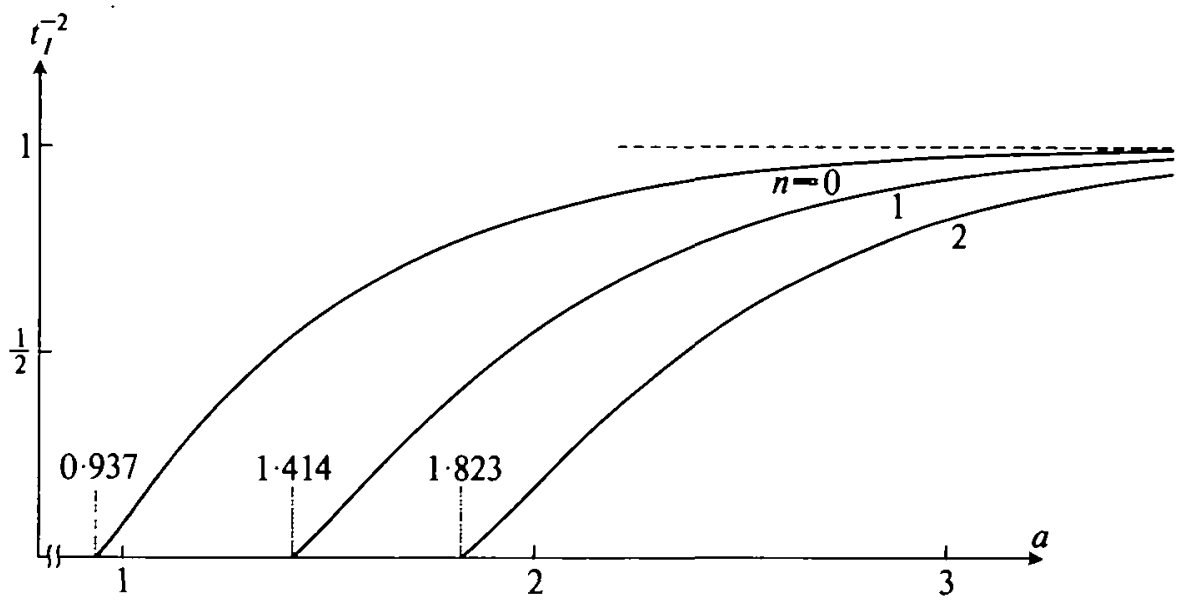

FIG. 7. Induction times $t_{\mathbf{I}}$ for supercritical symmetric containers

As $a$ increases beyond the value 3, the values of $\alpha_{I}$ can be seen to increase dramatically. In these circumstances the effects of conduction from the walls of the containing vessel over unit time (the time, very nearly, in which thermal runaway now occurs) introduce only a small spatial variation near the peak value of $\phi$. This would correspond to $\alpha_{I}$ being large in equation (49). For much of its early development $\phi$ is almost homogeneous

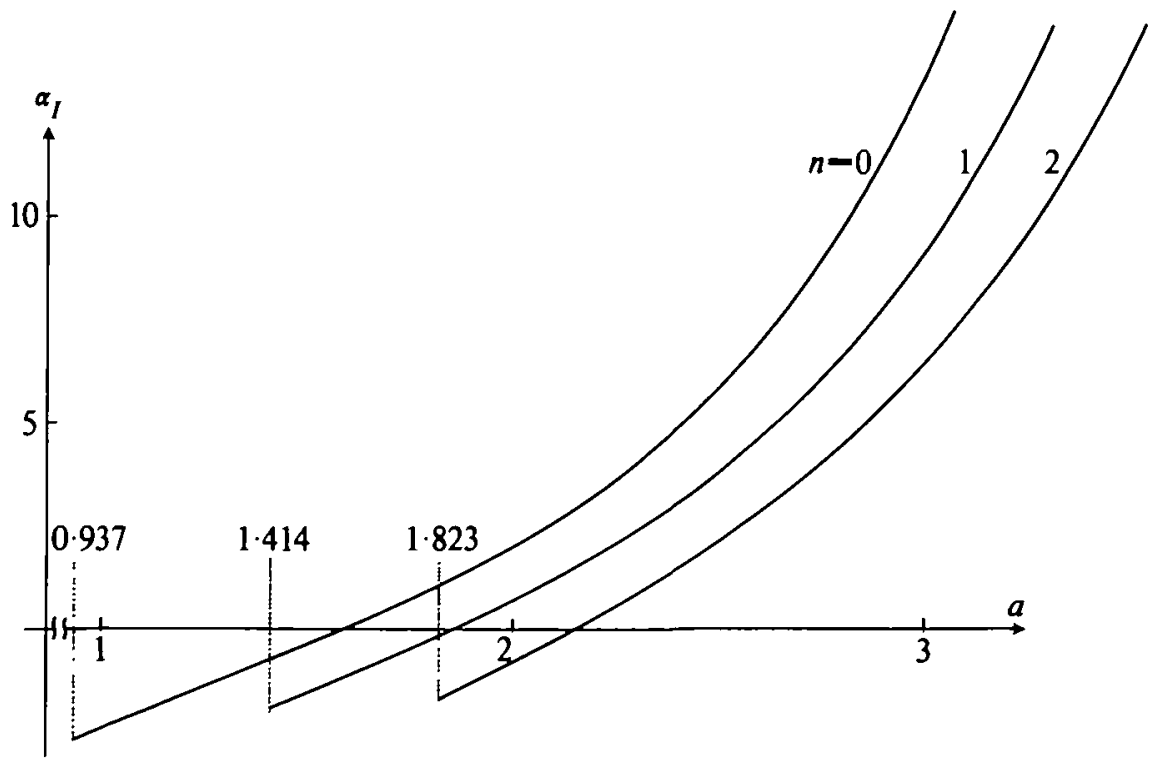

Fio. 8. Thermal runaway constants $\alpha_{l}$ for supercritical symmetric containers 


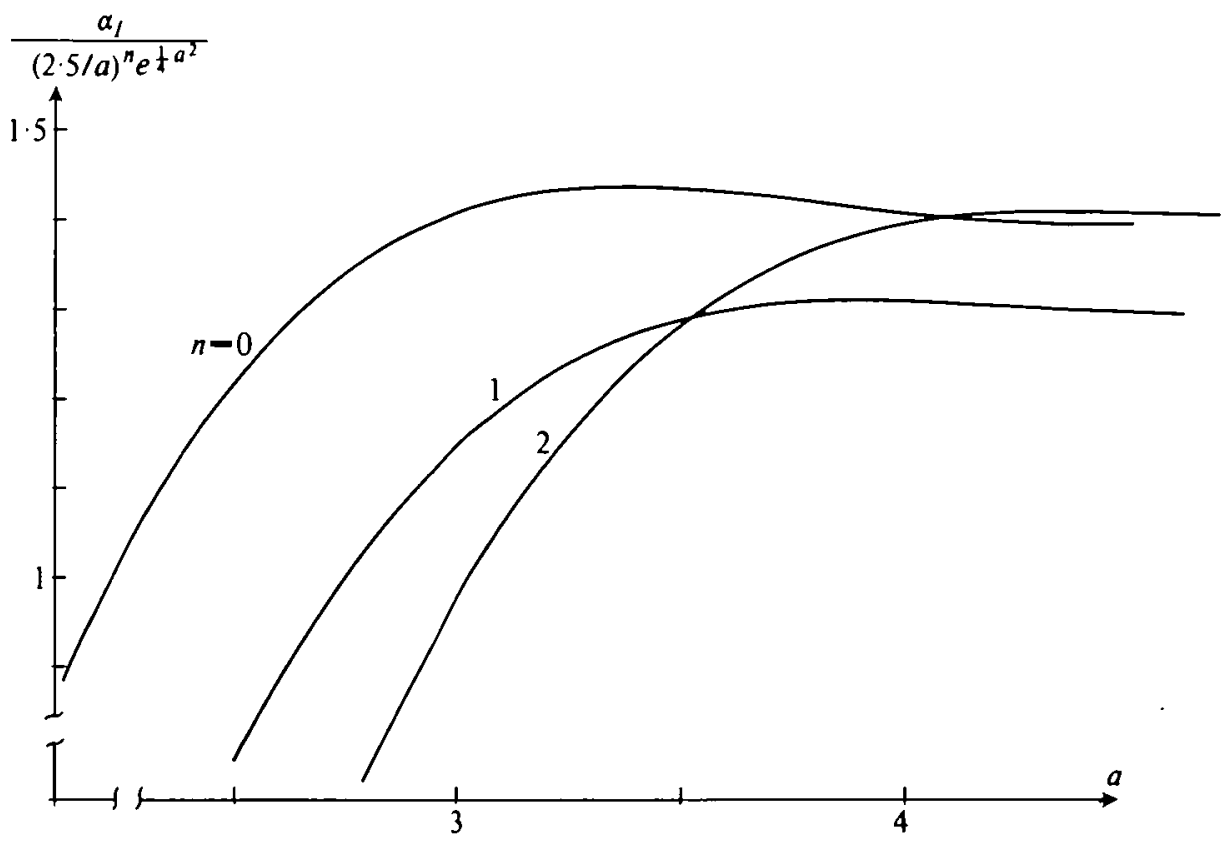

Fig. 9. Thermal runaway constants $\alpha_{I}$ for large containers

in the neighbourhood of $r=0$. It is found empirically from the numerical results that, as $a$ grows large, the values of $\alpha_{I}$ can be characterized in the form

$$
\alpha_{I} \approx\left(\frac{2 \cdot 5}{a}\right)^{n} e^{t a^{2}} \times \begin{cases}1 \cdot 4, & n=0, \\ 1 \cdot 3, & n=1, \\ 1 \cdot 4, & n=2\end{cases}
$$

for large values of $a$, as shown in Fig. 9.

Also as a grows large, the values of $t_{I}$ approach unity, this being the adiabatic homogeneous induction time (9). Being a measure of departure from homogeneity, one should expect $\alpha_{I}$ to be related to the difference $t_{I}-1$. This is so, as sketched in Fig. 10, in which it can be seen that $t_{I}$ varies like

$$
t_{I} \approx 1+(1+n) \alpha_{I}^{-3} \times 0.4
$$

for large values of $\alpha_{1}$. For near critical values of $a$ the induction times are large and can be seen from Fig. 7 to vary like $\left(a-a_{\mathrm{cr}}\right)^{-\frac{1}{1}}$.

\section{Behaviour outside the Ignition Kernel}

In order to examine the behaviour of $\phi$ at positions close to the induction position $r=r_{l}$, but not tending towards it as the induction time is ap- 


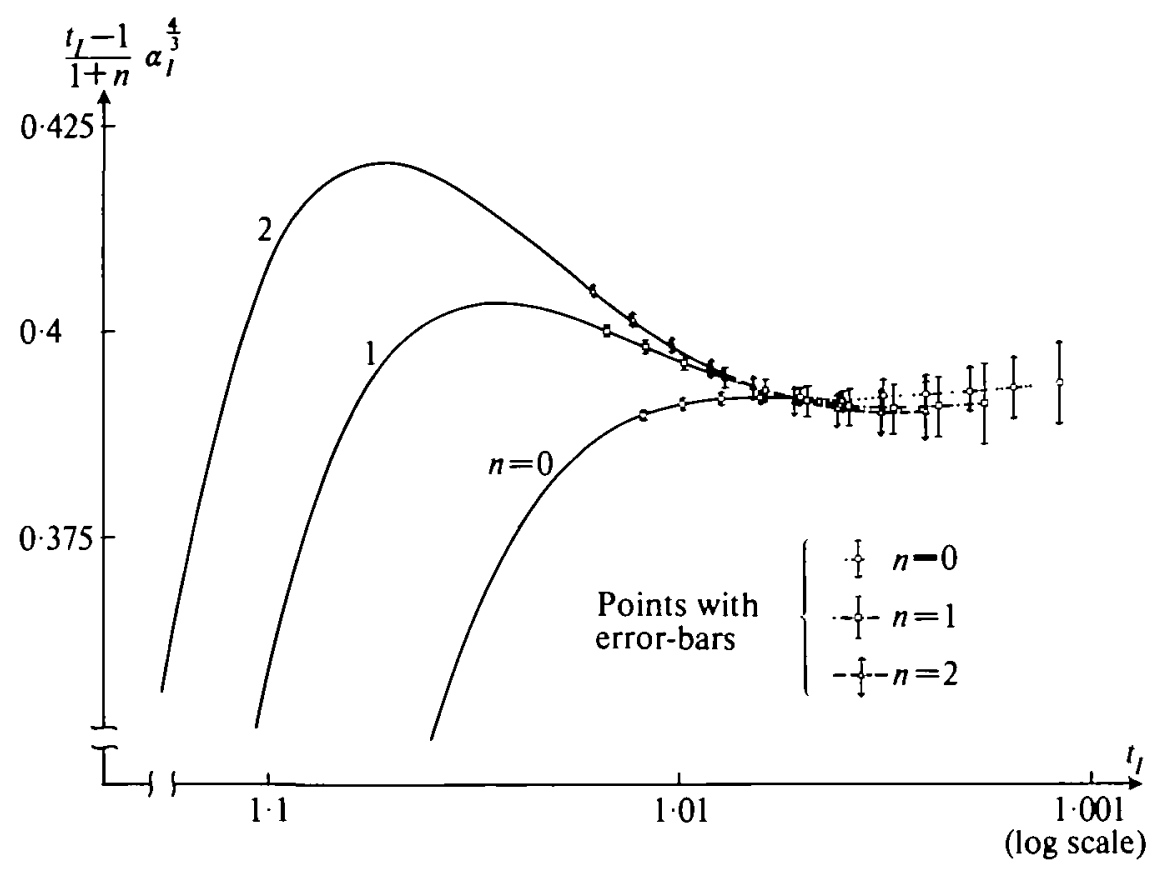

Fia. 10. Relation between induction times $t_{1}$ and thermal runaway constants $\alpha_{I}$ for large containers

proached, it is convenient to introduce a 'large' parameter $X^{\prime}$ ', and make the following rescalings of $r$ and $t$ :

$$
\left.\begin{array}{l}
\tau^{\prime} e^{-X^{\prime}}=-e^{-\zeta}=\frac{t-t_{I}}{e^{\alpha_{1}}}, \\
2 x\left[X^{\prime} e^{-X^{\prime}}\right]^{t}=\left[\zeta e^{-\zeta}\right]^{\sharp} \rho=\frac{r-r_{I}}{e^{\frac{1}{2} \alpha_{T}}} \cdot
\end{array}\right\}
$$

In terms of $x$ and $\tau^{\prime}$ it can be seen that $\phi$ satisfies the partial differential equation

$$
\phi_{\tau^{\prime}}=\frac{1}{4 X^{\prime}}\left[\phi_{x x}+\frac{n \cdot 2\left[X^{\prime} e^{\alpha_{1}-X^{\prime}}\right]^{\sharp}}{r_{\mathrm{I}}+2\left[X^{\prime} e^{\alpha_{1}-X^{\prime}}\right]^{\frac{\beta}{2} x}} \phi_{x}\right]+\lambda e^{\phi+\alpha_{1}+X^{\prime}} .
$$

When $\zeta$ is within order one of $X^{\prime}, \tau^{\prime}$ will be of order one, and order-one values of $\rho$ will correspond to order-one values of $x$. At this stage any solution for $\phi$ in terms of $x$ and $\tau^{\prime}$ must match with the asymptotic solution (49) for $\phi$ in terms of $\rho$ and $\zeta$. By simply substituting for $\rho$ and $\zeta$ in equation (49) and checking that the result is indeed an asymptotic solution of equation (57), it is readily seen that the required asymptotic solution for $\phi$ 
in the limit as $X^{\prime}$ tends to infinity with $x$ and $\tau^{\prime}$ fixed takes the form

$$
\begin{aligned}
\phi+\alpha_{I}= & X^{\prime}-\ln \left(x^{2}-\tau^{\prime}\right)-\frac{\ln X^{\prime}}{X^{\prime}} \frac{5+n_{I}}{2} \frac{x^{2}}{x^{2}-\tau^{\prime}}+ \\
& +X^{-1}\left[\frac{1+n_{I}}{2}-\frac{x^{2}}{x^{2}-\tau^{\prime}} \ln \left(x^{2}-\tau\right)\right]+O\left(\frac{\ln ^{2} X^{\prime}}{X^{\prime 2}}\right) .
\end{aligned}
$$

This solution extends the Ignition-Kernel solution (48) for $\phi$ in two ways. First, it describes the behaviour of $\dot{\phi}$ in regions left outside the Ignition Kernel at some stage as it shrinks towards $r_{I}$. Secondly, the solution is valid beyond the induction time $t_{\mathrm{I}}$ for non-zero values of $x$. Barring any other causes of breakdown, such as the passage of deflagration flames or significant effects due to thermal expansion, asymptotic breakdown would ultimately occur close to the line of singularity represented by

$$
\tau^{\prime}=x^{2} .
$$

The solution (58) is derived with $x$ taking the role of an independent variable while $X^{\prime}$ behaves as a large parametric constant in equation (57) and the definitions (56). The result is still valid, however, if it is considered to be in terms of a constant value of $x$, say $x=1$, with $X^{\prime}$ allowed to vary as a function of $r$, representing, for large values of $X^{\prime}$, a coordinateperturbation expansion of $r$ about $r_{I}$. In this way the solution can be expressed in the form

$$
\begin{aligned}
\phi+\alpha_{I}= & X-\ln (1-\tau)-\frac{\ln X}{X} \frac{5+n_{I}}{2} \frac{1}{1-\tau}+ \\
& +X^{-1}\left[\frac{1+n_{I}}{2}-\frac{1}{1-\tau} \ln (1-\tau)\right]+O\left(\frac{\ln ^{2} X}{X^{2}}\right),
\end{aligned}
$$

where

$$
\frac{r-r_{I}}{e^{\frac{1}{2 \alpha_{1}}}}=\frac{2 X^{\frac{1}{1}}}{e^{\frac{1 X}{x^{x}}}}, \quad \frac{t-t_{I}}{e^{\alpha_{1}}}=\frac{\tau}{e^{X}},
$$

and where the primes of $X^{\prime}$ and $\tau^{\prime}$ have been dropped because of the difference between these definitions and the definitions (56). In particular, at the induction time $(\tau=0)$, this gives the temperature profile

$$
\begin{aligned}
\phi\left(r, t_{I}\right)+\alpha_{I} & =X-\frac{5+n_{I}}{2} \frac{\ln X}{X}+\frac{1+n_{I}}{2 X}+O\left(\frac{\ln ^{2} X}{X^{2}}\right) \\
& =\ln [\omega \ln \omega]-\frac{3+n_{I}}{2} \frac{\ln \ln \omega}{\ln \omega}+\frac{1+n_{I}}{2 \ln \omega}+O\left(\frac{\ln ^{2} \ln \omega}{\ln ^{2} \omega}\right),
\end{aligned}
$$

where $\omega=4 e^{\alpha_{2}} /\left(r-r_{I}\right)^{2}$. The result expressed in terms of $\omega$ follows from substituting for $X$ in terms of $r$. By considering only order-one terms 
or greater, $\phi\left(r, t_{\mathrm{I}}\right)$ has the following asymptotic form:

$$
\phi\left(r, t_{I}\right)=\ln \left[\frac{4 \ln \left[4 /\left(r-r_{I}\right)^{2}\right]}{\left(r-r_{I}\right)^{2}}\right]+o(1) .
$$

Figure 11 presents a comparison of the asymptotic solution (61) with solutions for $\phi\left(r, t_{I}\right)$ obtained under the conditions described in the previous section, for the case in which $a=3$. The horizontal line would be the form of the Hot-Spot asymptotic solution (20) for the case when $n=0$, having $A=4.5$ (from (6)). The dotted line is the first-order asymptotic solution (62).

These results demonstrate that, by means of the intermediate expansion (60), the Ignition-Kernel solution does match, as it must do, with a solution for $\phi$ of the form (18). It is interesting to compare the asymptotic result (62)

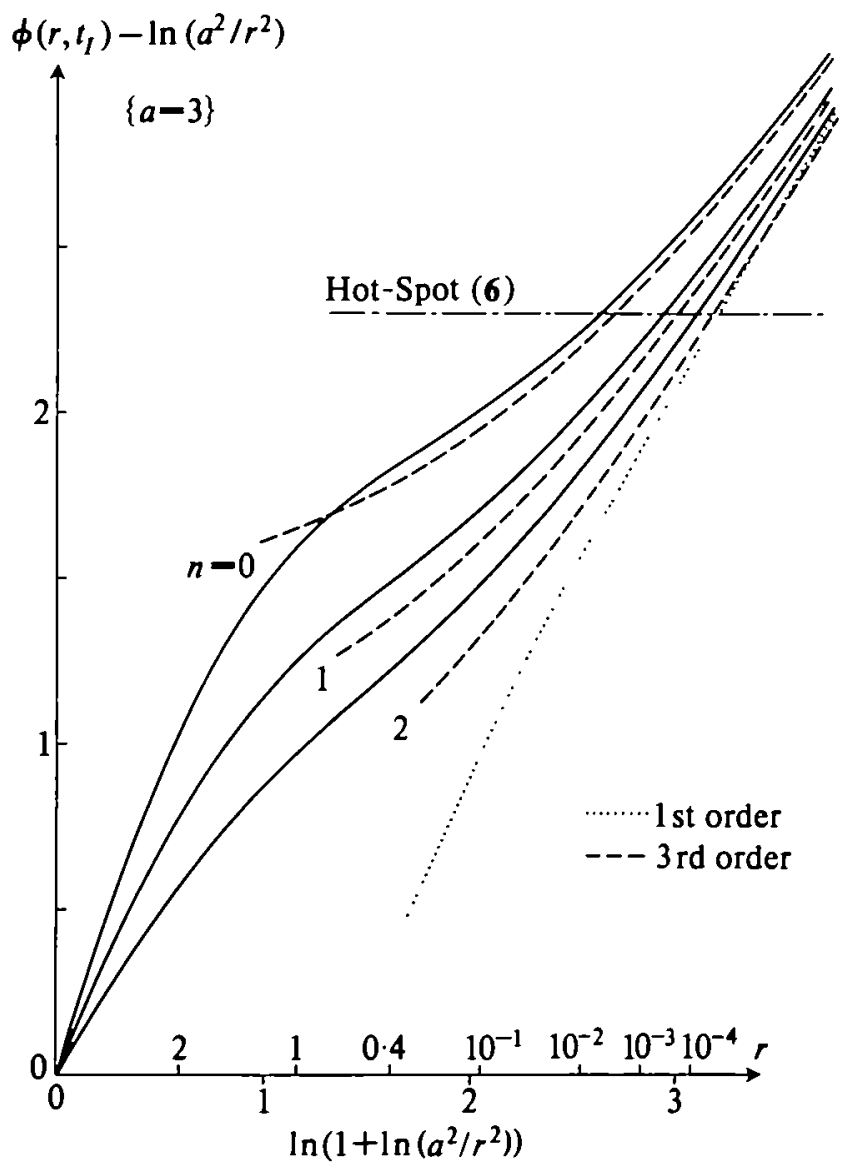

Fig. 11. Asymptotic and numerical temperature profiles at the induction time $t_{I}$ 
with equation (20) which would have been the corresponding result consistent with a valid Hot-Spot solution. As with all the results obtained in the Ignition-Kernel analysis, the asymptotic form (62) for $\phi$ is uniquely determined to first order.

Finally, the line of singularity of equation (60), beyond or within a neighbourhood of which no finite solution for $\phi$ is possible, is now given by

or

$$
\tau=1 \text {, }
$$

$$
\begin{aligned}
t & =t_{I}+\frac{\left(r-r_{I}\right)^{2}}{4 X} \\
& =t_{I}+\frac{\left(r-r_{I}\right)^{2}}{4} /\left\{\ln \left[\frac{4 \ln \left[4 /\left(r-r_{I}\right)^{2}\right]}{\left(r-r_{I}\right)^{2}}\right]+\alpha_{I}+o(1)\right\} .
\end{aligned}
$$

Although deflagration and thermal-expansion effects (arising from a fuller consideration of the combustion processes than is possible with equation (1)) may invalidate equation (60) before these times are reached, this result does reveal a constraint on the time available for such effects to emerge before the thermal-runaway process itself would drive the reaction towards its completion. A fuller elaboration of this point, with its implications for the nature of the ignition process, will be made in a subsequent paper (4).

\section{Conclusions}

As spatially varying thermal runaway develops it takes on a well-defined form:

$$
\phi \sim-\ln \left[t_{I}-t+\frac{\frac{1}{4}\left(r-r_{I}\right)^{2}}{\alpha-\ln \left(t_{I}-t\right)}\right],
$$

which is remarkably independent of both the conditions leading to thermal runaway and the topology of the thermal-runaway region. The asymptotic expansion, describing this development, is found to agree well with careful numerical solutions of the governing equation. It also reveals in its derivation the manner in which the nonlinearity of the problem and the effect of conduction conspire to impose such a well-defined form on the rapidly rising temperature profile.

An underlying structure in the thermal-runaway process is revealed, in which a strongly supercritical thermal runaway (where the relative effects of conduction are initially small) is found to be very much like a less strongly supercritical, but more highly-developed, thermal runaway. This development is characterized by the progressive diminution towards zero of the relative effect of conduction compared with the reaction rate near the peak temperature value; locally, the rate of self-heating accelerates dramatically while, in comparison, the conductive impediment to the temperature growth diminishes. 


\section{Acknowledgements}

The bulk of this work was carried out as part of a research programme sponsored by the SERC in the College of Aeronautics, Cranfield Institute of Technology, under the title 'The burning of finite quantities of gas' (SERC Contract B/RG/3662.2).

The author is grateful to J. F. Clarke for his invaluable assistance in the conduct of this research, and to D. R. Kassoy for stimulating an important part of the work.

\section{REFERENCES}

1. D. A. Frank-Kamenetski, Acta Physicochimica U.R.S.S. 16 (1942) 357.

2. - Diffusion and Heat Exchange in Chemical Kinetics (University Press, Princeton, 1955).

3. J. W. Dold and J. F. Clarke, Ignition and Criticality of a Finite Quantity of Gas Released in the Atmosphere, 20th International Combustion Symposium, Michigan, 1984.

4. - 'Ignition by thermal runaway', preprint, University of Bristol.

5. A. K. KAPILA, SIAM J. appl. Math. 39 (1980) 21.

6. D. R. Kassoy and J. Poland, ibid. 39 (1980) 412.

7. J. W. Doun, 'The auto-ignition and combustion of a finite region of gaseous fuel (Ph.D. Thesis, Cranfield Institute of Technology, 1979).

8. A. A. LACEY, IMA J. appl. Math. 27 (1981) 71.

9. D. R. Kassoy, Comb. Sci. Tech. 10 (1974) 27.

10. J. D. BuCKMASTER and G. S. S. LUDFORD, Theory of Laminar Flames (University Press, Cambridge, 1982), 232-247. 